\title{
Physics Needs for Future Accelerators
}

\author{
J. D. Lykken \\ Theoretical Physics Dept. \\ Fermi National Accelerator Laboratory \\ Batavia, IL 60510
}

\section{Prologomena to any meta future physics}

The title assigned to this talk, while certainly both eye-catching and pithy, doesn't make any literal sense in English. "Physics needs for future accelerators" is a phrase which could be interpreted in any number of ways, leading to very different sorts of talks. Let me begin, therefore, by briefly describing the roads not taken in my review. This will allow me to mention some important issues which, while not the focus of my talk, are worthy of serious high-minded discussion in international forums such as the Lepton-Photon meetings.

\subsection{Physics needs for building future accelerators}

This, it seems to me, is the most obvious literal interpretation of the original title. Since, like all theorists, I consider myself to be essentially omniscient, I even briefly considered giving such a talk. I went as far as dusting off my copy of Jackson [1], which contains, after all, most of the basic physics that you will need to build future accelerators.

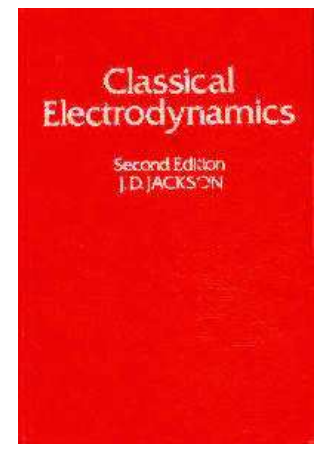

Figure 1: The book. 
This facetious exercise illustrates a worrisome development to which we had better start paying more attention. Since (in principle) all of us understand the basics of

accelerator physics, there is a disturbing tendency to denigrate this essential branch of our field, to relegate it to "technicians" - the implication being that first-rate minds are drawn to more "cutting-edge" physics problems. Combined with the general trend towards increasing specialization, we have largely decoupled and discounted an activity which in fact largely defines and limits the future of our field.

Furthermore, if you look seriously at the designs and R\&D work related to any of the proposed future accelerators -linear colliders [2, 3], hadron colliders [4], as well as very ambitious ideas like the muon collider [5], or the CLIC two-beam acceleration concept [6]-you will find a host of interesting and highly challenging physics problems. As a community, I doubt that we are doing enough towards attracting, training, supporting, and encouraging the next generation of accelerator physicists -the cadre of first-rate, creative, and experienced people without whom no future accelerators are likely to get built. In that case everything else I say in this talk addresses a moot topic, since we will have failed before we have even properly begun.

How can we avoid this calamity? One way to attract new talent to accelerator $\mathrm{R} \& \mathrm{D}$, and to validate the importance of this activity, is for more experimentalists -and even theorists - to devote some finite fraction of their time to thinking about accelerator physics problems. We should keep in mind that lack of technical experience has the positive virtue of stimulating new kinds of questions, new ways of thinking, and thus ultimately to innovation. Bringing fresh minds to bear on these sorts of problems can only help, and will enhance the prestige, and thus the overall vigor, of these activities. Small steps in this direction can be encouraged now with existing resources; a few years down the road we should aim to establish new centers for advanced accelerator physics.

The payoff for nuturing this branch of physics will be well worth the investment, and could be spectacular. It may well be, for example, that physics innovation, rather than engineering or manufacturing breakthroughs, is the key to major cost reductions in linear collider or hadron collider designs.

\subsection{Physics needs for funding future accelerators}

Another possible interpretation of the title of this talk is a little more political. What do we need to get out of existing experiments and facilities in order to strengthen the case for particular future machines, as well as goose up the overall momentum and glamour of our field?

While we are correct to spend sleepness nights agonizing over the optimal path to our long term future, we should also rejoice that our immediate future looks extremely bright. As this transparency from the next Lepton-Photon conference illustrates, the LEP experiments have a very real chance of discovering the Higgs. The B factories 


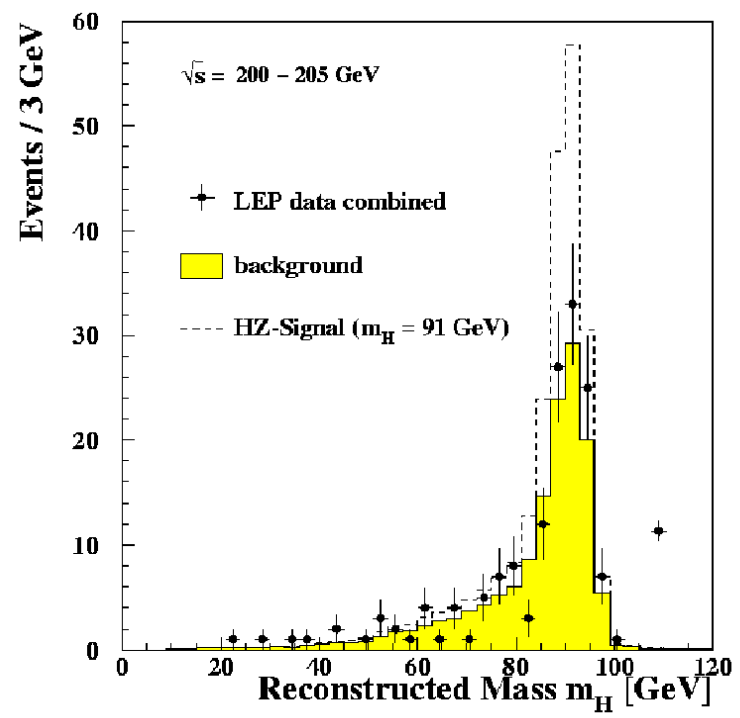

Figure 2: A plot from LP01?

coming on line now will provide fundamental new inputs to our global view of particle physics, including, most likely, some surprises. The same can be said for the many neutrino experiments in progress or under construction, and for a number of other low energy projects. As a bonus, a flood of new astrophysical data will impact on a number of important ideas and problems circulating in particle physics.

Last but not least, the next run of the Tevatron will extend our reach for the Higgs [7], supersymmetry [8], B physics, top physics, electroweak physics, and new strong dynamics, to name but a few. Major discoveries are very possible. If we are fortunate, we might even get the first experimental hints of extra spatial dimensions $[9$, 10], quantum gravity, or strings [11, 12, 13].

Obviously new discoveries from any of these arenas will help bootstrap funding and resources for future experiments and new facilities. In addition, discoveries -or even hints- of physics beyond the Standard Model will crystalize our thinking about what future facilities are needed.

We can congratulate ourselves on having assembled such a rich physics program for our immediate future. There is a danger, however, that in our rush to get to the LHC and other future accelerators, we may not maximally exploit those opportunities already at hand. If we are so foolish as to sacrifice our present to "secure" our future, we are more likely to end up sacrificing both. 


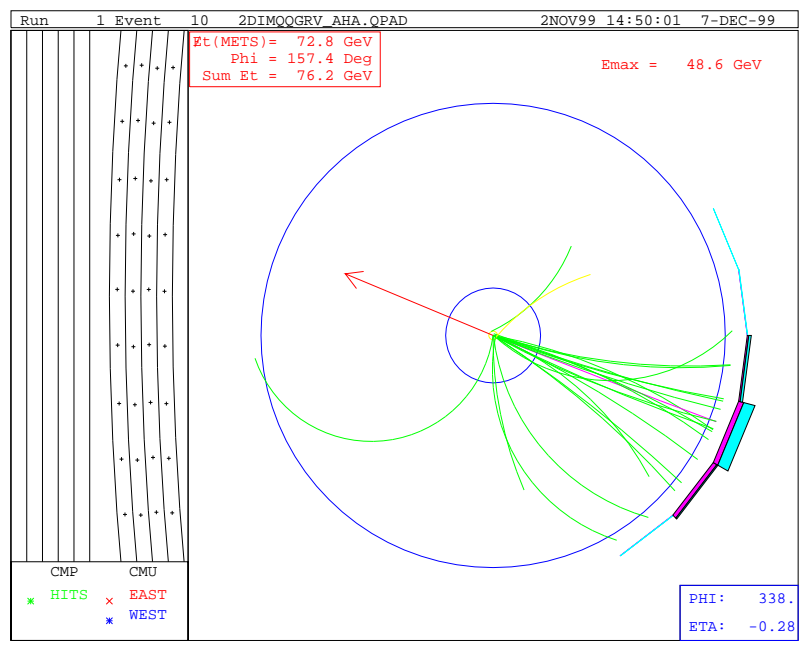

Figure 3: Extra dimensions at the Tevatron: a monojet recoiling off a Kaluza-Klein graviton. Simulated event from M. Spiropulu, CDF.

\section{Physics questions for future accelerators}

What is the right way to think about future physics? The traditional approach is to enumerate a number of possible scenarios for physics beyond the Standard Model, guided by whatever theoretical prejudices seem most fashionable at the moment. Although I will myself be guilty of this sort of theorizing later in the talk, I don't think this is the best approach for making major decisions, decisions that will commit billions of dollars of the taxpayers' money and determine the future or nonfuture of high energy physics.

I will attempt instead to outline a more robust approach to thinking about future physics, one which relies less on theoretical assumptions and more on an appreciation for what high energy physics is really all about.

\subsection{Crimes and misapprehensions}

The first thing we need to do is to discredit some harmful dogmas which have been clouding our thinking about high energy physics for more than a decade. Anyone who falls under the spell of these dogmas is not likely to hold rational or constructive views about the future needs of our field. As far as I know, no one is willing to admit authorship of these pernicious doctrines, but certainly the bourgeois overlords of high energy physics and their running dog lackeys (i.e. this audience) must assume responsibility for tolerating or promulgating these ideas.

The basic crime here is to have allowed the misapprehension -among ourselves, 
students, and the public- that particle physics is "almost done". This misapprehension arises from two rather different but equally radical notions, which I will now briefly review.

\subsubsection{Organized religion}

This is the dogma that high energy physics has been to a large extent supplanted by a new activity called string theory. String theory is the one true Theory of Everything (TOE), people who are a lot smarter than you will have it figured out any day now, and they will soon be able to compute the electron mass, etc. purely on the basis of mathematical consistency. Thus the traditional activities of high energy physics (such as experiment) have become largely irrelevant. Put another way, since no future accelerator can ever directly probe the most fundamental scale of physics, the "bottom up" approach is pointless, and we should instead invest in the more promising "top down" approach to connecting fundamental physics with our existing data banks.

This extreme view is, of course, quite silly. String theory does indeed hold great promise for advancing our understanding of fundamental physics, and has already produced some profound insights about black hole physics, about gauge theories, and other areas as well. But anyone who has followed the rapid advances in string theory knows that, for every question successfully disposed of, three new ones seem to crop up in its wake. Fundamental physics is, not surprisingly, rich, dense, and confusing. The road to fundamental understanding will be a long road, and this makes the traditional activities of high energy physics (such as experiment) even more interesting and important in that light.

\subsubsection{Feudalism}

This is the dogma that the Standard Model is king and will reign forever. This is particularly discouraging for young people, because the implication here is that all the good stuff happened in the seventies and you missed it. There are a few things left to do -we'll find the Higgs, measure a few more parameters- and then that's it. So unfortunately young physicists entering the field today are coming in at the tail end of the Golden Age, but -tough luck- there's only one Golden Age and ours is almost over.

This extreme view is equally silly, yet it seems to have penetrated into the morose

subconscious of a large fraction of high energy physicists. If you are suffering from this problem and the Zoloft isn't working, pay attention and I will attempt to dispel your weltschmerz by rational argument. 


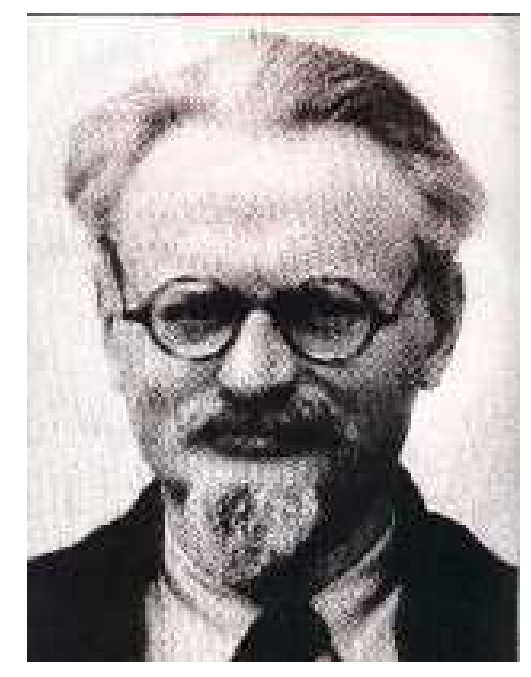

Figure 4: Why is this man smiling?

\subsubsection{Trotsky was right}

As it turns out, somewhat surprisingly, the guy who had it right was Trotsky. This great philosopher and humanist was the first to point out that high energy physics is exciting, and will continue to remain exciting, precisely because it exists in a state of permanent revolution. As experiments probe higher energies and smaller distance scales, and as our theoretical frameworks struggle to produce a coherent explanation of what we see, our fundamental view of the physical world and how to describe it changes dramatically. High energy physicists are continually engaged in a process of creating new frontiers, moving to those frontiers, civilizing the rough elements, then pushing out to the next frontier.

This rather obvious historical fact has lately been obscured by two phenomena. One phenomenon is that particle physics during the past decade was in one of those recurring phases where theoretical assimilation of earlier experimental findings dominates (to some extent) over new experimental surprises. This gives the superficial impression that not much is happening, when in fact during this period our understanding has increased dramatically, such that our basic theoretical frameworks are remarkably different from what they were 10 or 15 years ago.

The second phenomenon was the cancellation of the SSC, and the concomitant popularization of the high energy physics version of Horganism. The idea here is that we have slipped into an era of diminishing returns for our investments in basic science. High energy physics, in this view, is becoming increasingly expensive and complicated to pursue, and as a practical matter the field will die out completely in 
a decade or two.

This dire prediction may indeed be correct, but for reasons rooted in politics and sociology, not physics. Precisely because high energy physics is constantly redefining itself, we have no idea what it will be like a century from now. Advances of the 21st century could easily rival those of the 20th century. There is no reason to imagine that we are near the end of this process, barring the complete collapse of our civilization. Or, as Trotsky put it, our expectation is

\section{Revolution whose every successive stage is rooted in the preced- ing one and which can end only in complete liquidation.}

\subsection{The Standard Model as an effective field theory}

Let me now be much more explicit about the present status of particle physics, and about the process of getting to the next iteration of our understanding. The key realization here is that the Standard Model is an effective field theory. As advocated in Ken Wilson's pioneering work [14], quantum field theories in general are nonperturbatively defined as effective field theories valid below some explicit ultraviolet cutoff $\Lambda$. The parameters of the effective Hamiltonian can be regarded as encoding the effects of integrating out the ultraviolet degrees of freedom above the cutoff scale, including the high momentum modes of the light degrees of freedom. Effective Hamiltonians can contain an arbitrarily large number of operators, but at energies small compared to the cutoff the effects of higher dimension operators are suppressed by powers of energy divided by $\Lambda$.

The Standard Model, of course, is famously renormalizable. However, as emphasized by Steven Weinberg [15], the power-counting renormalizability of the Standard Model has no particular physical significance. The physically important sense in which the Standard Model is renormalizable is that the ultraviolet divergences of the model are controlled by gauge symmetries, such that counterterms exist to cancel all infinities. In this sense effective gauge field theories with higher dimension operators are also renormalizable. Renormalizability is in no sense an indication that the Standard Model is "fundamental". Our generic expectation is that the full Standard Model Hamiltonian will turn out to contain a number of higher dimension operators, whose effects are too suppressed to have shown up in present experiments. Indeed it is this expectation which makes precision low energy experiments interesting as probes of new physics.

In light of the above, the enterprise of searching for new physics can be described in a completely model-independent way. The task for experiments at future accelerators (as well as at existing facilities) is to address the following set of physics questions:

- The Standard Model is an effective field theory for physics below some high energy cutoff $\Lambda$. What is the value of $\Lambda$ ? 
- What are the relevant degrees of freedom in the new effective theory at energies above $\Lambda$.

- What are the symmetries and organizing principles of this new effective theory?

- What symmetries and organizing principles of the Standard Model turn out to be artifacts of the "low energy" approximation?

- Do the symmetries and organizing principles of the new effective theory explain the parameters and parameter hierarchies of the Standard Model (e.g. all the notorious mysteries of flavor)?

- Does the new effective theory give any hints (e.g. higher dimension operators, spontaneously broken symmetries) of new physics at even higher scales?

\subsection{What is the scale of new physics?}

Let me elaborate further on the first of these questions, which has obvious importance for making smart choices about future accelerators. How do we go about determining $\Lambda$ for the Standard Model? There seem to be three basic complementary methods.

- One method is to use high energy machines to search for evidence of new degrees of freedom characteristic of the new effective theory above the cutoff. This could take the form of new particles, resonances, or collective effects. It could also show up as evidence of compositeness, form factors, indications of symmetry restoration, or of symmetry breaking. We might even see signals telling us about new spatial degrees of freedom.

So far all such searches have turned up negative, even at the Tevatron, our highest energy collider. This indicates either that the new degrees of freedom are somewhat obscured (by Standard Model backgrounds and instrumental effects) or that present experiments are not yet probing above the cutoff scale $\Lambda$.

- Another method is to search for evidence of higher dimension operators in the effective Hamiltonian of the Standard Model itself. At high energies this approach is not entirely distinct from the previous method, but it can also be utilized in a variety of lower energy experiments. It is important to realize here that the symmetries and approximate symmetries of the known contributions to the Standard Model may not be respected by the full effective action. This motivates a variety of searches for flavor changing neutral currents, CP violation, lepton number violation, proton decay, etc.

So far, with the exception of the strong case for neutrino oscillations, experiments of this type have not produced compelling results for new physics. This 


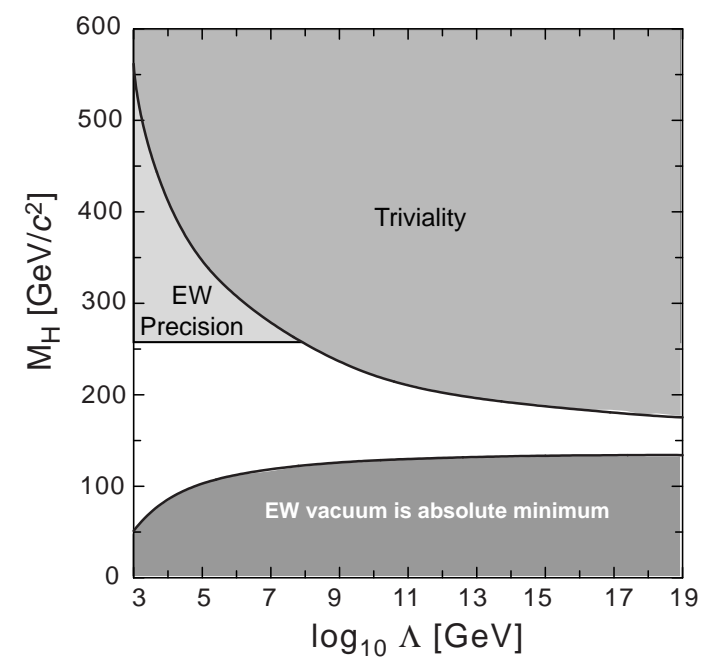

Figure 5: The Higgs mass and upper bounds on the cutoff scale. From C. Quigg, hep-ph/9905369.

is an indication either that certain operators are simply forbidden in the full effective action, or that $\Lambda$ is sufficiently large that these "irrelevant" operators decouple rather efficiently in current experiments.

- The third method is to consider the Higgs sector, which is more sensitive to $\Lambda$ for several reasons. The first is that, in the case where the Higgs mass is large, the Higgs becomes strongly coupled at some high energy scale. $\Lambda$ is certainly no larger than this scale. Similarly, in the case where the Higgs mass is small, the Higgs effective potential becomes unstable at some high energy scale, also providing an upper bound on $\Lambda$. These limits are summarized in Figure 5, where we see that they have only logarithmic sensitivity to the high scale. Also shown is the current 95\% confidence level upper bound on the Standard Model Higgs mass from electroweak precision data, which is approximately $250 \mathrm{GeV}$. Combined with the lower bound on the Higgs mass from the direct searches at LEP, the net result is that $\Lambda$ is rather weakly bounded.

A much stronger and more robust upper bound on $\Lambda$ is provided by consideration of the Higgs naturalness problem. The Higgs has an unprotected scalar mass, whose value is naturally of order the cutoff $\Lambda$. This would lead us to the conclusion that $\Lambda$ not much more than $250 \mathrm{GeV}$. A light Higgs can be arranged if, in the new effective theory above $\Lambda$, the Higgs mass is related to parameters which vanish in some symmetry limit. However even in these cases it is hard to arrange a large hierarchy between the electroweak scale (defined as the Higgs 
vacuum expectation value $v=246 \mathrm{GeV}$ ) and the cutoff $\Lambda$.

The bottom line of this effective field theory analysis is that there is a strong (and growing) tension in the Standard Model between the Higgs naturalness/hierarchy problem, which wants $\Lambda$ to be close to the electroweak scale, and the apparent decoupling of new physics effects in current data. The obvious resolution of this dialectic is that $\Lambda$ is only just out of reach of current experiments. An educated guess would be:

$$
500 \mathrm{GeV} \lesssim \Lambda \lesssim 1 \mathrm{TeV}
$$

I emphasize that although the numbers in this estimate are a little soft, the physics input that goes into it is very robust. Indeed it ultimately only depends on our understanding of the Standard Model as an effective quantum field theory.

\subsection{What could be out there?}

What will we find when we begin to probe the new effective theory above the scale $\Lambda$ ? Most theoretical speculation about the new effective theory at high energies involves adding things to the Standard Model:

- Add new particles: 4th generation, superpartners, messenger sector, etc.

- Add new symmetries: e.g. supersymmetry, etc.

- Add new gauge interactions: e.g. technicolor, $Z^{\prime}$, etc.

However it is just as likely that at higher energy scales we have instead (or in addition) much more radical changes:

- Qualitatively new degrees of freedom: e.g. strings, membranes, extra dimensions.

- Symmetries of the Standard Model are broken: e.g. B and L violation.

- "Sacred principles" of the Standard Model are violated! This would not be the first time that sacred cows got ground into hamburger.

Indeed, already in this century we have seen several examples of sacred principles which turned out to be artifacts of some approximation. It is worth reminding ourselves of these history lessons:

1. Newtonian mechanics $\Leftrightarrow$ electromagnetism $\rightarrow$ special relativity.

Lesson: Galilean invariance is only an approximation, good at low speeds. 
2. Thermodynamics $\Leftrightarrow$ electromagnetism $\rightarrow$ quantum mechanics.

Lesson: Rayleigh's formula for blackbody emittance is only an approximation, good at low frequencies.

3. Newtonian gravity $\Leftrightarrow$ special relativity $\rightarrow$ general relativity.

Lesson: Newtonian gravity is only an approximation, good for weak gravitational fields and low speeds.

A conservative view is that all of the following theoretical assumptions/frameworks may break down under certain conditions at certain energy scales:

- The assumption that the fundamental dynamical entities are point-like particles.

- Relativistic quantum field theory (and the associated ideas of locality, microcausality, CPT invariance).

- General relativity.

- Quantum mechanics.

It is unfortunate that these possibilities are today largely ignored by both theorists and experimenters. Of course we should not ascribe every burp in the data to a breakdown of microcausality, but neither should we assume that all of our current paradigms will remain sacrosanct indefinitely. It is amusing to recall in this regard that Werner Heisenberg, in 1939, seriously suggested that quantum mechanics breaks down at an energy scale around $1 \mathrm{GeV}$. Nowadays anyone who questions the universal validity of quantum mechanics is (usually correctly) labelled as a crank.

Although string theory has not (yet) done a good job of matching to the Standard Model at low energies, it has proven to be a great exercise for both organizing and liberating our thinking about new physics. For example:

- If string theory is correct, both general relativity and quantum field theory break down at some energy scale $M_{s}$. We don't know what this string scale is; it's lower bound, set by experiment, is about $1 \mathrm{TeV}$.

- If string theory is correct, the fundamental physical entities are not quarks and leptons, but a whole collection of particle-like, string-like, and membrane-like objects.

- Furthermore these objects propagate in a $9+1$ or $10+1$ dimensional spacetime. 


\subsection{Model-independent conclusions}

Without making any particular assumptions about what new physics is out there, we can now draw some important conclusions with a high degree of confidence:

- There is a whole new effective theory waiting to be explored at the TeV scale.

- The new physics will be rich, surprising, confusing, and take a long time to untangle.

These conclusions also imply the following:

- To explore the new theory you will want high energies, reasonable luminosities, and reasonable detectors.

- To understand the new physics, you will also want detailed studies, for which you need excellent luminosities and excellent detectors.

- You will need detailed studies not only to unravel the new effective theory, but also to give you hints about physics at even higher scales.

\section{Future accelerators}

In the second half of my talk I would like to discuss the physics driving various proposals for future accelerators. To be concrete and focus our thinking I have summarized these proposals below according to my opinion of what we might have and when. The dates of course are only estimates, but the groupings are important. It is important in thinking about future machines to make a clear distinction between those which are intended to operate in the LHC era, and those which are clearly post-LHC successors. It is also important to discriminate between those proposals which extend the energy frontier, and those which would operate within the energy frontier defined by the LHC. I have also made note of possible upgrades of the Tevatron, LHC, and linear $e^{+} e^{-}$collider (LC), since recycling is likely to become increasingly popular in a difficult funding climate.

Given the very exciting prospect for future physics discoveries advocated in the first half of this talk, it is rather straightforward to make the physics case for machines of the LHC era. I emphasis that my summary of the physics needs does not factor in the dollar cost or political cost of various machines, neither do I address the question of whether we can attract sufficient human resources to pursue several big projects simultaneously. I will also touch briefly on machines of the post-LHC era. 
2006 - 2012: The LHC Era

- LHC: $\sqrt{s}=14 \mathrm{TeV}, \mathcal{L}=10^{33}$ to $10^{34}$.

- LC: $\sqrt{s}=350 \mathrm{GeV}$ to $1 \mathrm{TeV}, \mathcal{L}=10^{34}$ ish.

- $\nu$ factory: 1 millimole of muons per year.

- upgraded Tevatron?: $\sqrt{s}=4-6 \mathrm{TeV}$.

\section{3 - 2025: Within the Energy Frontier}

- stretch LC: $\sqrt{s}=1.5 \mathrm{TeV}$.

- $\gamma \gamma, e^{-} e^{-}$: piggyback on LC.

- First Muon Collider: Higgs factory? Heavy Higgs factory?

\section{3 - 2025: Extending the Energy Frontier}

- upgraded LHC?: $\sqrt{s}=$ ?

- CLIC: $\sqrt{s}=3-5 \mathrm{TeV}, \mathcal{L}=10^{35}$.

- High Energy Muon Collider: $\sqrt{s}=3-4 \mathrm{TeV}$, potential for 10 to $15 \mathrm{TeV}$.

- VLHC: $\sqrt{s}=100-200 \mathrm{TeV}, \mathcal{L}=10^{35}$.

\subsection{What is the physics driving the LHC?}

You are supposed to know this already! The LHC will advance the energy frontier by roughly a factor of five over present experiments. This should be amply sufficient to probe the new effective theory above the cutoff $\Lambda$, discover most of its degrees of freedom, symmetries, and organizing principles. Looking back, we should be able to get a fundamental understanding of the mechanism of electroweak symmetry breaking, and perhaps shed light on flavor problems or other mysteries of the Standard Model.

\subsection{What is the physics driving the LC?}

The various linear collider proposals involve machines which will operate within the energy frontier established by the LHC. I have attempted here to summarize the important physics needs driving these proposals:

- Higgs physics is golden.

- The LHC won't be sufficient to unravel the new physics at the TeV scale.

- The LC has unique capabilities to divine new physics at even higher scales. 


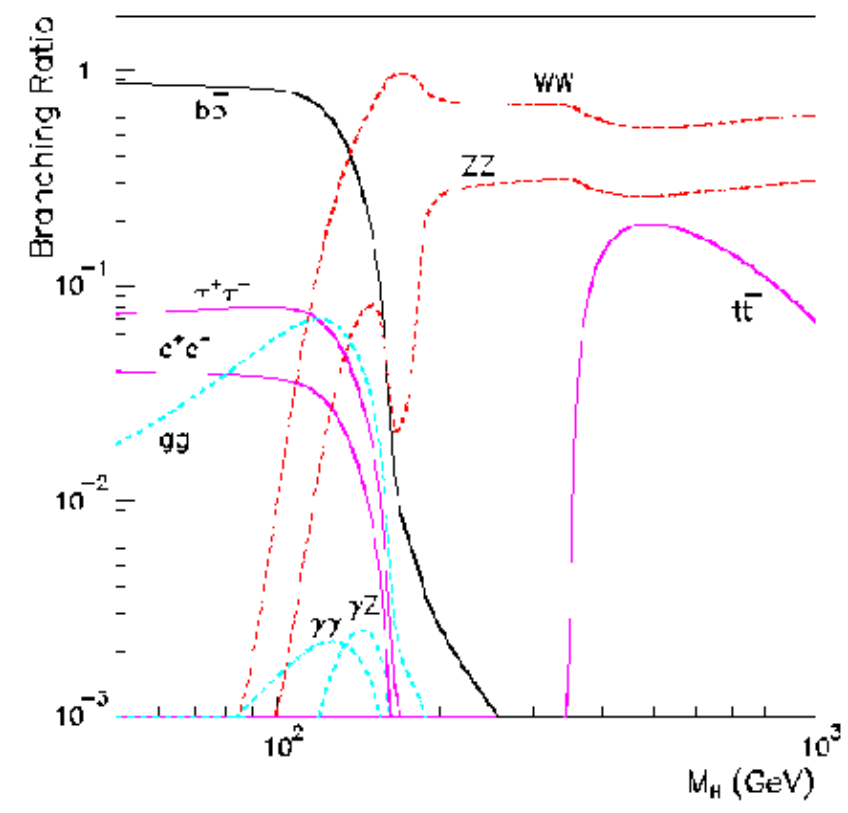

Figure 6: Branching fractions versus mass for a Standard Model Higgs.

\subsubsection{Higgs physics is golden}

Precision measurements are already telling us that either the Higgs is light (mass less than about $250 \mathrm{GeV}$ [16]), or new physics is misleading us! Even if the Higgs turns out not to be Standard Model-like, it is still likely to be discovered at either LEP, the Tevatron, or the LHC. However, the discovery of a $b \bar{b}$ invariant mass bump (say) in some data sample will be only the beginning of understanding the Higgs. As soon as a discovery is made, a main priority of high energy physics will be to answer the following questions [17]:

1. Is this the Higgs of electroweak symmetry breaking?

2. Is this the Higgs associated with the generation of fermion masses?

3. Is this the only Higgs?

In short, we will need to find out everything we possibly can about the Higgs sector. Higgs physics will be golden, will occupy us for many years, and will require a large number of challenging measurements.

For example, we will need precise measurements of all the Higgs branching fractions. Disentangling the Higgs branching to taus, charm, and glue is a tall order, and 
we will need sensitivity to the rare decay modes as well. The LHC can do part of this job, but we will need an LC (with good luminosity and detectors) to do the rest. For a light Higgs a 350 or $500 \mathrm{GeV}$ linear collider has enough energy reach. Eventually we would want to look at $t \bar{t} H$ production at higher energies.

Higgs physics will be interesting for a long time. Thus even in the post-LHC era we may be very interested in low energy machines which can refine our knowledge of the Higgs sector. This includes the $\gamma \gamma$ option for a linear collider, and an s-channel Higgs factory as First Muon Collider.

\subsubsection{The LHC won't be sufficient to unravel the new physics at the TeV scale.}

The LHC experiments will do a lot, including a large number of precision measurements. But, as I have argued, the new physics at the $\mathrm{TeV}$ scale will be both rich and confusing. Mere prudence will demand that we probe this new world with all the tools at our disposal. A linear collider offers different sensitivities, polarization, reduced backgrounds, better contained events, and even more precise measurements. Examples:

- Untangling the neutralino and slepton sectors in supersymmetry. What variety of SUSY is it?

- Deciphering virtual effects of extra dimensions. Is your Drell-Yan anomaly due to spin 2 Kaluza-Klein graviton exchange?

\subsubsection{LC precision measurements can pin down new physics scales}

A case study which illustrates this point was recently made by Ambrosanio and Blair [18]. They assumed that the new physics is a minimal version of gauge-mediated supersymmetry, and examined the question of whether experiments at a $500 \mathrm{GeV}$

linear collider could measure the hidden sector supersymmetry breaking scale $\sqrt{F}$.

This is a scenario in which the lightest neutralino is the next-to-lightest-superpartner (NLSP), and decays to a Goldstino (which is not seen) plus a photon: $\tilde{\chi}_{1}^{0} \rightarrow \gamma \mathrm{G}$. The decay length of the NLSP, $c \tau$, has only log sensitivity to the gauge mediation messenger scale, but is proportional to the SUSY breaking scale $\sqrt{F}$ :

$$
c \tau_{\tilde{\chi}_{1}^{0}} \sim \frac{F^{2}}{M_{\tilde{\chi}_{1}^{0}}^{5}}
$$

This suggests a challenging physics program, in which we must first determine that we have weak scale supersymmetry, that we have gauge-mediated supersymmetry breaking, that this is "minimal" gauge mediation, and that the neutralino is the 
NLSP. Having done all of this, we must then be prepared to measure $c \tau$ of $\tilde{\chi}_{1}^{0}$ in the entire range from 10 microns to 30 meters, using various (overlapping) techniques:

- Projective tracking

- 3D tracking

- Photon pointing

- Calorimeter timing

- Statistical (counting single $\gamma$ versus $2 \gamma$ )

The conclusion of this study is that in such a scenario, with an appropriate detector and $200 \mathrm{fb}^{-1}$ of integrated luminosity, we could measure $\sqrt{F}$ to $\pm 5 \%$ at a $500 \mathrm{GeV}$ linear collider.

\subsection{Why a Neutrino Factory?}

Neutrino oscillations are a strong hint that there is new physics associated with scales in the $10^{10}-10^{16} \mathrm{GeV}$ range, or of brane-bulk physics in the case of extra dimensions [19]. It will be a big job to pin down this new physics, and, in particular, to link this new physics to anything else in the Standard Model We will certainly need precise and overconstrained measurements of the lepton mass matrix, just as we are now achieving for the CKM matrix of the quark sector. Flavor problems are hard, and it seems highly probable that we will need to build at least one new accelerator facility optimized for neutrino physics.

Possible designs [20] of a muon storage ring neutrino factory are currently under study [21, 22]. the basic is to use a muon storage ring as a source for very intense beams of fairly high energy neutrinos. The muon charge, momentum, and polarization determine the neutrino composition and spectrum, thus the initial characteristics of the neutrino beam will be known with high confidence. Many types of oscillation experiments can be considered [23], including very long baseline possibilities such as Fermilab to Gran Sasso or CERN to Brookhaven. This presents the opportunity for truely international collaborations. This is particularly so since there are serious detector challenges to be overcome, including building in the capability to discriminate lepton flavors and measure their charges.

My belief that the neutrino factory can be brought on line as an LHC era facility is based mostly on its moderate size, and upon the growing interest and enthusiasm exhibited in many quarters for this idea. However a number of serious technical obstacles stand in the way of this goal, and without an especially aggressive R\&D effort my rosy scenario will not come to pass. Since a neutrino factory appears also to be the most practical path to a muon collider, we have an especially strong motivation to pursue this idea as vigorously as possible. 


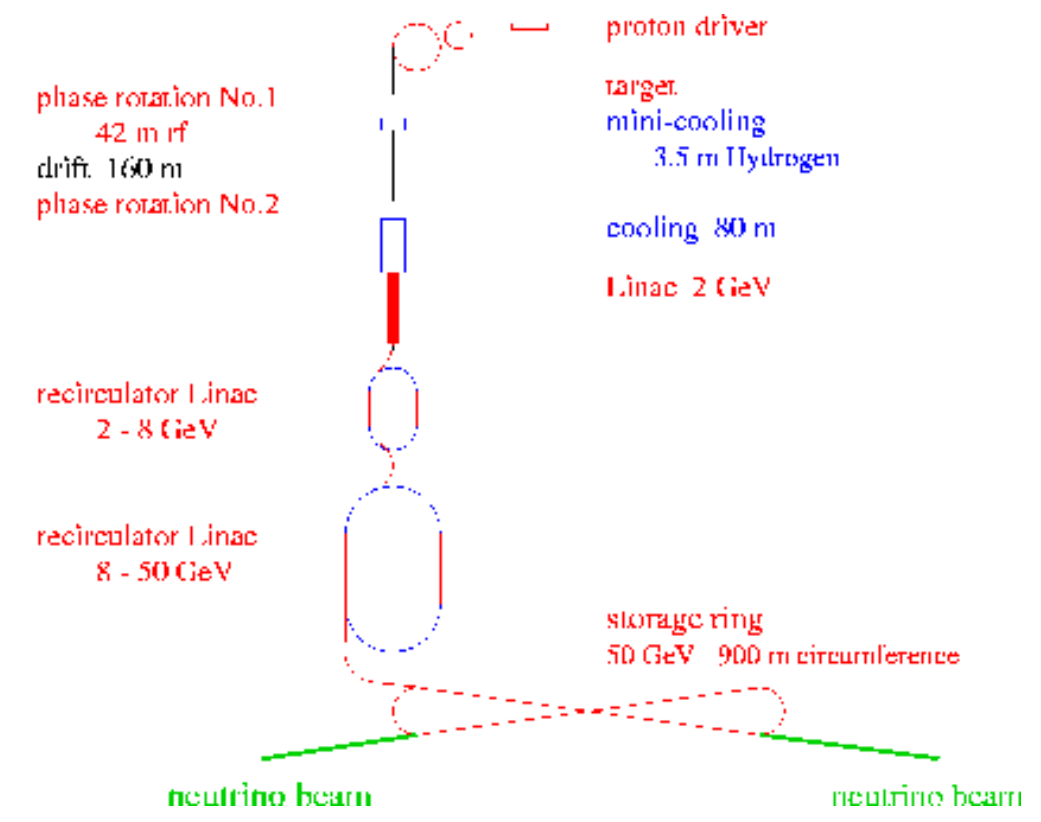

Figure 7: Schematic for a muon storage ring neutrino factory.

\subsection{Pushing the energy frontier}

The reality of modern high energy physics is such that, if you want a new energy frontier collider in 2020, you had better be doing serious R\&D for it now. This puts us in something of a quandry, since we don't yet know how to estimate the next interesting energy scale. From the arguments reviewed in this talk we will certainly need a post-LHC energy frontier machine, but beyond that general statement we know almost nothing at this point in time.

In fact at the moment we cannot with confidence answer even the most basic of questions. For example, to explore the new physics of the post-LHC era, will a 3 or $4 \mathrm{TeV}$ lepton collider, or an LHC upgrade, be good enough, or do we need to push straight to a 10-15 TeV muon collider or 100-200 TeV VLHC? Until we answer such questions we had better be pursuing $\mathrm{R} \& \mathrm{D}$ for all options.

The point here is that $\mathrm{LHC}+\mathrm{LC}$ data is going to be essential for making good decisions about post-LHC facilities. For example, LHC/LC data may indicate that, due to the existence of extra dimensions, the effective Planck scale is only about $3 \mathrm{TeV}$. In this case hard scatterings at future colliders may produce mostly black holes. This knowledge would certainly have a major impact on your choices for $\sqrt{s}$, luminosity, and detector design for post-LHC experiments! 


\section{A Final Thought}

It is much more likely that we will fail to build new accelerators than that these accelerators will fail to find interesting physics!

\section{References}

[1] J. D. Jackson, Classical Electrodynamics, 2nd Edition, John Wiley \& Sons, 1975.

[2] B. H. Wiik, "The TESLA Project", Part. Accel. 62, 43 (1998).

[3] "Zeroth Order Design Report for the Next Linear Collider", SLAC-R-0474, May 1996.

[4] G. Anderson et al, "Summary of the Very Large Hadron Collider Physics and Detector Workshop", hep-ph/9710254.

[5] C. Ankenbrandt et al, "Status of Muon Collider Research and Development and Future Plans", Fermilab-Pub-98-179, Phys. Rev.ST Accel.Beams 2081001 (1999).

[6] J-P Delahaye et al, "CLIC, a $0.5 \mathrm{TeV}$ to $5 \mathrm{TeV} e^{+} e^{-}$Compact Linear Collider", CERN/PS 99-005, CERN/PS 99-062, Acta Phys. Polon. B30, 2029 (1999).

[7] Draft report of the "Physics at Run II" Higgs Working Group, available at http://fnth37.fnal.gov/higgs.html.

[8] Draft reports of the "Physics at Run II" Supersymmetry/Higgs Workshop, available at http://fnth37.fnal.gov/susy.html.

[9] N. Arkani-Hamed, S. Dimopoulos, and G. Dvali, Phys. Rev. D59 086004 (1999).

[10] J. Lykken and L. Randall, "The Shape of Gravity", hep-th/9908076.

[11] J. Lykken, Phys. Rev. D54 3693 (1996).

[12] E. Accomando, I. Antoniadis, and K. Benakli, "Looking for TeV Scale Strings and Extra Dimensions", hep-ph/9912287.

[13] S. Cullen, M. Perelstein, and M. Peskin, "TeV Strings and Collider Probes of Large Extra Dimensions", hep-ph/0001166.

[14] K. G. Wilson, Rev. Mod. Phys. 55583 (1983).

[15] S. Weinberg, The Quantum Theory of Fields, Vol. II, Cambridge University Press, 1996. 
[16] The LEP Electroweak Working Group, http://www.cern.ch/LEPEWWG].

[17] M. Carena, S. Mrenna, and C. E. M. Wagner, "The Complementarity of LEP, the Tevatron, and the LHC in the Search for a Light MSSM Higgs", hep-ph/9907422.

[18] S. Ambrosanio and G. Blair, "Measuring Gauge Mediated Supersymmetry Breaking at a $500 \mathrm{GeV} e^{+} e^{-}$Linear Collider", hep-ph/9905403.

[19] N. Arkani-Hamed, S. Dimopoulos, and G. Dvali, "Neutrino Masses from Large Extra Dimensions", hep-ph/9811448.

[20] S. Geer, C. Johnstone, D. Neuffer, "Design Concepts for a Muon Storage Ring Neutrino Source", Fermilab-Pub-99-121.

[21] FNAL Feasibility Study on a Neutrino Source Based on a Muon Storage Ring, http://www.fnal.gov/projects/muon_collider/nu-factory/nu-factory.htm].

[22] B. Autin, A. Blondel, and J. Ellis (eds), "Prospective Study of Muon Storage Rings at CERN", CERN-99-02, ECFA 99-197.

[23] V. Barger, S. Geer, K. Whisnant, "Long Baseline Neutrino Physics with a Muon Storage Ring Neutrino Source", hep-ph/9906487. 